\title{
La bibliothèque de Saint-Just : catalogue et essai d'interprétation critique
}

Louise Ampilova-Tuil, Catherine Gosselin et Anne Quennedey

\section{(2) OpenEdition \\ Journals}

Édition électronique

URL : https://journals.openedition.org/ahrf/13454

DOI : $10.4000 / a h r f .13454$

ISSN : 1952-403X

Éditeur :

Armand Colin, Société des études robespierristes

Édition imprimée

Date de publication : 15 février 2015

Pagination : 203-222

ISBN : 978-2-200-92958-9

ISSN : 0003-4436

Référence électronique

Louise Ampilova-Tuil, Catherine Gosselin et Anne Quennedey, « La bibliothèque de Saint-Just : catalogue et essai d'interprétation critique », Annales historiques de la Révolution française [En ligne], 379 | janvier-mars 2015, mis en ligne le 15 février 2018, consulté le 01 juillet 2021. URL : http:// journals.openedition.org/ahrf/13454 ; DOI : https://doi.org/10.4000/ahrf.13454 


\title{
LA BIBLIOTHÈQUE DE SAINT-JUST : CATALOGUE ET ESSAI D'INTERPRÉTATION CRITIQUE
}

\author{
Louise AMPILOVA-TUIL, Catherine GOSSELIN et Anne QUENNEDEY
}

Mis sous scellés avec ses papiers au soir du 9 Thermidor ${ }^{1}$, les livres de Saint-Just furent inventoriés le 13 fructidor an III (30 août 1795) après leur transfert au dépôt littéraire de la rue Cerutti. Cet inventaire ${ }^{2}$ a attiré à plusieurs reprises l'attention des chercheurs : à la fin du XIX $\mathrm{X}^{\mathrm{e}}$ siècle, Germain Bapst ${ }^{3}$ en a donné une transcription accompagnée de remarques et, plus récemment, Agnès Marcetteau-Paul et Dominique Varry ${ }^{4}$ lui ont consacré un développement dans leur étude d'un corpus de vingt-six inventaires de bibliothèques de la période révolutionnaire. Ce sont dans les deux cas des commentaires brefs (moins d'une page) rendant seulement compte d'une partie des livres répertoriés par l'inventaire, en sorte que l'on ne dispose pas d'une analyse de la bibliothèque de Saint-Just qui soit exhaustive et suffisamment précise.

Cet article pose les bases d'une telle étude critique. On trouvera d'abord une nouvelle transcription de l'inventaire qui corrige les erreurs des transcriptions existantes ${ }^{5}$. Nous l'avons fait suivre du catalogue des trente-deux ouvrages que l'inventaire permet d'identifier, dans lequel nous indiquons chaque fois que possible l'édition que possédait Saint-Just. Il a

(1) AN, F/7/4775 11 .

(2) AN, F/17/1198, pièce ${ }^{\circ} 117$.

(3) « Inventaire des bibliothèques de quatre condamnés », La Révolution française, t. 21, juillet-décembre 1891, p. 535.

(4) Agnès MARCETTEAU-PaUl et Dominique VARRY, « Les bibliothèques de quelques acteurs de la Révolution, de Louis XVI à Robespierre », Livre et Révolution, Mélanges de la Sorbonne, $\mathrm{n}^{\circ} 9$, 1989, p. 204.

(5) Outre celle de Germain Bapst, il s'agit de la transcription faite par Michel Morineau en annexe d'un article sur Couthon, qui a le mérite d'être complète mais qui présente des erreurs empêchant d'identifier plusieurs livres (« Documents sur Georges Couthon », AHRF, n 253, 1983, p. 468-469). Une transcription manuscrite très lisible mais fautive est également conservée à la Bibliothèque nationale de France sous la cote NAF 22995. 
en effet paru que, dans la mesure où au XVIII ${ }^{\mathrm{e}}$ siècle le texte d'un ouvrage varie souvent considérablement d'une édition à l'autre, donner simplement une liste de titres et d'auteurs n'était pas satisfaisant : les éditions ont donc été identifiées en utilisant les ouvrages bibliographiques spécialisés, les catalogues des bibliothèques publiques et des librairies anciennes ainsi que les sources et les possibilités de recherche inédites qu'offre le réseau Internet.

À partir de ce catalogue, nous avons réalisé un premier essai d'examen critique qui prend en compte l'ensemble des livres répertoriés en les classant par domaines de connaissance ${ }^{6}$. Un développement sur le devenir des livres de Saint-Just conclut ce travail.

\section{Transcription du document $F / 17 / 1198$ (pièce $n^{\circ} 117$ ) des Archives nationales $^{7}$}

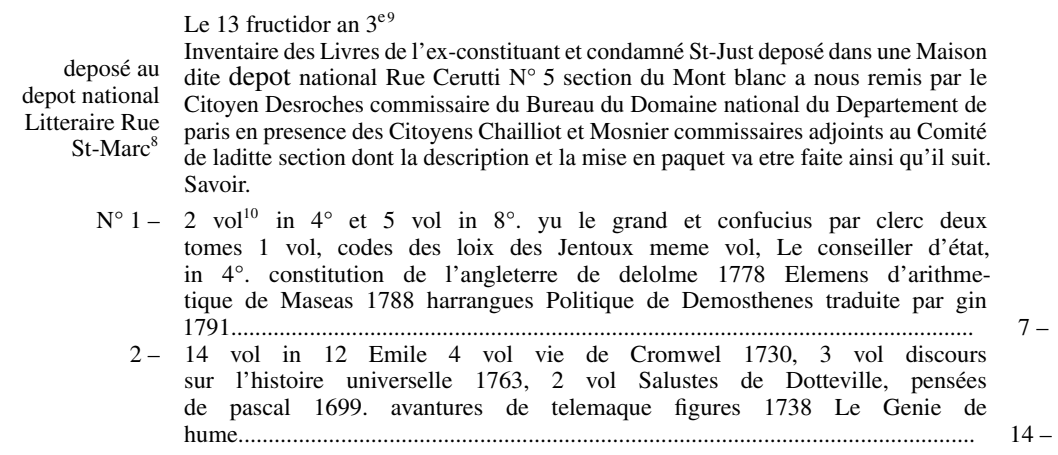

(6) Nous avons préféré ce mode de classement à la classification traditionnelle de la librairie parisienne selon cinq catégories (Belles-lettres, Histoire, Sciences et arts, Droit et jurisprudence, Religion) qui, en règle générale, est mal adaptée aux bibliothèques de la fin du XVIII ${ }^{\mathrm{e}}$ siècle. Notre jugement concorde avec celui de Claude Jolly, qui a aussi adopté une classification moderne dans son étude des livres de l'abbé Grégoire (« La bibliothèque de l'abbé Grégoire », Livre et Révolution, op. cit., p. 209-220).

(7) Notre transcription conserve la graphie, souvent fautive, et la ponctuation originales. Les inscriptions « $\mathrm{N}^{\circ} 1$ », « 2 », etc. de la première colonne de l'inventaire renvoient aux numéros des paquets de livres. Les nombres de la troisième colonne correspondent au nombre de volumes contenus dans chaque paquet («7 - », par exemple, se lit « 7 volumes »).

(8) Inscription postérieure (Cf. en fin d'article la section sur le devenir des livres de Saint-Just après fructidor an III).

(9) Cette date, qui paraît avoir été écrite postérieurement, comporte des surcharges qui la rendent difficilement déchiffrable. Il est en effet également possible de lire non pas « 13 fructidor an $3^{\mathrm{e}}$ » mais « 12 fructidor an $3^{\mathrm{e}}$ », cette dernière date correspondant à celle de la vente des autres biens de Saint-Just rue Caumartin.

(10) Dans tout l'inventaire, « vol » abrège « volume[s]». 
3 - 14 vol uvres de Montesquieu 6 vol, Du meme le tôme 4. Revolutions Romaine les tomes 1 et 3 . principes de Moral de Moral [sic] de Mably formant le tome 15 de ses uvres, Les autres ne meritant description.

4 - $16 \mathrm{vol}$ in $12 \mathrm{rel}^{11}$ et Broches complets et incomplets oraisons choisies de Ciceron latine et françaises 2 vol Caracteres de Theophrastes 1750, 2 vol Uvres posthumes du chevalier Temple, histoire poetique 1777. fables de la fontaine 1788 Contrat social 1791. Lettres d'une peruvienne 1787 pensées de J. J. Rousseau Brochés 2 vol Gerusalem delivrée 1793 figure 2 vol Manuel des Jeunes Republicains Brochés.

5 - 16 vol in $8^{\circ}$. Brochés Code Militaire 17933 vol Recueil de pieces affaires du temps 2 vol, confessions de J. J. Rousseau tomes 3e Les chaines de l'esclavage Memoire sur l'education des bêtes a laine par Duquesnoy les autres affaires du temps ne meritant aucune description, Plus les Nos 20 a 25 des tableaux de la Revolution francaise 6 Cahiers.

\section{Catalogue succinct des ouvrages mentionnés dans l'inventaire}

La liste suivante ${ }^{12}$ a été réalisée en croisant les indications fournies par l'inventaire (dates d'édition, formats des livres, nombre de volumes) avec les ressources informatisées du Catalogue collectif de France qui répertorie les livres conservés à la Bibliothèque nationale de France et dans les principales bibliothèques municipales et universitaires françaises, les catalogues en ligne de plusieurs grandes bibliothèques publiques étrangères ${ }^{13}$ ainsi que les catalogues de vente de librairies spécialisées en livres anciens. Les bibliographies des éditions de Montesquieu et de Rousseau ont également été consultées ${ }^{14}$. Nous avons parfois pu identifier certains livres rares en nous aidant de Google Books (Google Livres) malgré les difficultés d'utilisation de cette bibliothèque numérique pour la recherche bibliographique ${ }^{15}$.

Les ouvrages sont rangés par ordre d'inscription dans l'inventaire. Pour chacun des ouvrages, la graphie et la ponctuation des titres et sous-titres sont données d'après la page de titre.

(11) « rel » est mis pour « reliés ».

(12) On trouvera des précisions sur ces ouvrages et leurs auteurs dans : Anne QUENNEDEY, Un sublime moderne : l'éloquence de Saint-Just à la Convention nationale, thèse, Paris-Sorbonne, 2013, t. III, p. 147-193.

(13) En particulier la Bibliothèque cantonale et universitaire de Lausanne et la bibliothèque de l'Université d'Ottawa.

(14) Jean ERHARD, « Les Euvres complètes de Montesquieu », La Notion d'Euvres complètes, Studies on Voltaire and the eighteenth century, $\mathrm{n}^{\circ} 370,1999$, p. 49-55 ; Théophile DuFOUR, Recherches bibliographiques sur les æuvres imprimées de J.-J. Rousseau, Paris, L. Giraud-Badin, 1925 ; Jean SÉNELIER, Bibliographie générale des œuvres de J.-J. Rousseau, Paris, PUF, 1950.

(15) Les notices des ouvrages mis en ligne sur Google Books sont notoirement insuffisantes et très inférieures à celles réalisées pour les bibliothèques traditionnelles. Pour notre recherche, nous avons particulièrement eu à regretter que ne soient spécifiés ni le format des livres scannés ni les bibliothèques qui les conservent. Cependant, il a souvent été possible de remédier à ces défauts grâce aux indications de catalogage portées sur les pages et couvertures des livres, qui ont permis de remonter aux catalogues des bibliothèques concernées. 
1. Clerc, Nicolas Gabriel. Yu le Grand et Confucius, histoire chinoise. Soissons, Ponce Courtois, 1769,1 vol. in- $4^{\circ}$.

2. Halhed, Nathaniel Brassey. Code des loix des Gentoux, ou Réglemens des Brames, traduit de l'Anglois, d'après les Versions faites de l'original écrit en Langue Samskrete. Paris, Stoupe, 1778, 1 vol. in-4.

3. Le Conseiller d'Estat, ou Recueil des plus generales considerations servant au maniment des affaires publiques. Divisé en deux parties. En la première est traicté de l'establissement d'un Estat. En la seconde, Des moyens de le conserver \& l'accroistre. Première édition : Paris, Estienne Richer, 1632, 1 vol. in- $4^{\circ}$; rééditions dans ce format par le même éditeur en 1633 et à Paris chez Michel Bobin en 1645.

4. Lolme, Jean Louis de. Constitution de l'Angleterre, ou Etat du gouvernement anglois, comparé avec la forme républicaine et avec les autres monarchies de l'Europe. Amsterdam, E. van Harrevelt, 1778, 1 vol. in $-8^{\circ}$.

5. Mazéas, Jean Mathurin. Elémens d'arithmétique, d'algebre et de géométrie, avec une Introduction aux Sections coniques. Ouvrage utile pour disposer à l'étude de la Physique, \& des Sciences Physico-Mathématiques. Paris, Nyon, 1788,1 vol. in- $8^{\circ}$.

6. Les Harangues politiques de Démosthène avec les deux harangues De la Couronne. Traduction nouvelle par M. Gin. Suivies de notes relatives aux circonstances présentes et des extraits de plusieurs comédies d'Aristophane. Paris, Didot fils aîné - Bossange, 1791, 2 vol. in- $8^{\circ 16}$.

7. Rousseau, Jean-Jacques. Émile ou de l'Éducation. Première édition : Amsterdam, Jean Néaulme, 1762, 4 vol. in-12 ; plusieurs rééditions en quatre volumes au même format jusqu'en 1794.

8. Leti, Gregorio. La Vie d'Olivier Cromwel. Amsterdam, Henri Desbordes, 1730, 3 vol. in-12.

9. Bossuet, Jacques Bénigne. Discours sur l'histoire universelle à Monseigneur le Dauphin : pour expliquer la suite de la Religion \& les changements des Empires. Paris, Christophe David, 1763, 2 vol. in-12. 
10. Traduction de Salluste avec La vie de cet historien et des notes critiques; Seconde édition revue, corrigée \& augmentée du Texte Latin à côté de la Traduction, avec des Variantes choisies; par Jean-Henri Dotteville, de l'Oratoire. Paris, Auguste Martin Lottin, 1763, 1 vol. in-12 ; plusieurs rééditions en un volume et au même format sont parues jusqu'en 1794.

11. Pascal, Blaise. Pensées de M. Pascal sur la religion, et sur quelques autres sujets. Edition nouvelle. Augmentée de beaucoup de Pensées, de la Vie de l'Autheur, \& de quelques Dissertations. Amsterdam, Henri Wetstein, 1699, 1 vol. in-12.

12. Fénelon, François de Salignac de La Mothe. Les Avantures de Télémaque, fils d'Ulysse. Nouvelle édition conforme au Manuscrit original, \& enrichie de Figures en taille douce. Amsterdam, J. Wetstein, G. Smith et Zacharie Chatelain, Rotterdam, Jean Hofhout, 1738, 1 vol. in-12.

13. Hume, David. Le génie de M. Hume, ou Analyse de ses ouvrages, dans laquelle on pourra prendre une idée exacte des Mours, des Usages, des Coutumes, des Loix, \& du Gouvernement du Peuple Anglois. Londres, se trouve à Paris chez Vincent, 1770, 1 vol. in-12.

14. Montesquieu, Charles Louis de Secondat baron de La Brède et de. Euvres de Montesquieu, 6 volumes. Il s'agit de l'une des éditions en 6 volumes in-12 ou in-16, ou encore de l'une des éditions en 7 volumes in-12 parues avant 1795 à laquelle manquerait un volume (ce qui expliquerait la présence du tome 4 des Euvres de Montesquieu mentionné juste après dans l'inventaire : cf. 15 ci-dessous).

15. Montesquieu, Charles Louis de Secondat baron de La Brède et de. Euvres de Montesquieu, tome IV. Ce volume isolé provient de l'une des éditions des Euvres en 6 ou 7 volumes in-12 ou in-16 (cf. ci-dessus) ou de l'une des éditions en 4 ou 5 volumes in- $8^{\circ}$ parues jusqu'en 1794.

16. Vertot, René Aubert de. Histoire des révolutions arrivées dans le gouvernement de la république romaine, tomes I et III. Première édition : Paris, François Barois, 1719, 3 vol. in-12 ; plusieurs rééditions au même format et en trois volumes jusqu'en 1794.

17. Mably, Gabriel Bonnot de. Euvres complètes de l'abbé de Mably, tome XV. Toulouse, N.-Etienne Sens, et Nîmes, J. Gaude, 1791, 1 vol. in-12 ; réédition chez les mêmes et dans un format identique en 1793. 
18. Oraisons choisies de Cicéron, latines et françoises. Traduction nouvelle. Deux éditions possibles : Lyon, Jacques Guerrier, 1723-1726, 2 vol. in-12 ; Lyon, Antoine Molin l'aîné, 1726, 2 vol. in-12.

19. La Bruyère, Jean de. Les Caractères de Théophraste, avec les Caractères ou Les Mours de ce siècle. Nouvelle Edition augmentée de quelques Notes sur ces deux Ouvrages, \& de la Défense de La Bruyère, \& de ses Caractères, par M. Coste. Paris, Michel-Etienne David, 1750, 2 vol. in-12.

20. Temple, William. CEuvres postumes du chevalier Temple, Contenant I. Un Essai sur les Mécontentemens populaires. II. Un Essai sur la Santé \& la longue Vie. III. Une défense de l'Essai sur le Savoir des Anciens \& des Modernes. Des Pensées sur les diferens états de la Vie \& de la Fortune, \& sur la Conversation. Utrecht, Guillaume van de Water, 1704, 1 vol. in-12.

21. Histoire poétique, tirée des poètes français; avec un dictionnaire poétique. Troisième édition, revue \& corrigée. Paris, Joseph Barbou, 1777, 1 vol. in-12.

22. La Fontaine, Jean de. Fables choisies, mises en vers par monsieur De La Fontaine ; avec un nouveau commentaire par M. Coste. Paris, JeanFrançois Bassompierre, 1788, 1 vol. in-12.

23. Rousseau, Jean-Jacques. Du Contrat social, ou Principes $d u$ Droit politique. Trois éditions possibles : Paris, Cazin, 1791, 1 vol. in-12 ; Strasbourg, Imprimerie de la Société typographique, 1791, 1 vol. in-12 ; Paris, Defer de Maisonneuve, 1791, 1 vol. in-12 $2^{17}$.

24. Graffigny, Françoise de. Lettres d'une Péruvienne. Lyon, Bruyset frères, 1787, 1 vol. in-12.

25. Rousseau, Jean-Jacques. Les Pensées de J. J. Rousseau, Citoyen de Genève. Première édition en deux volumes in-12: Amsterdam (Paris), Prault, 1766 ; plusieurs rééditions au même format et en deux volumes jusqu'en 1794.

26. Le Tasse, Torquato Tasso dit. Jérusalem délivrée, poëme du Tasse. Londres, s. n., 1793, 2 vol. in-12.

(17) Cette dernière édition comprend également les Considérations sur le gouvernement de Pologne et sur sa réformation projettée. 
27. Le Manuel des jeunes Républicains, ou Éléments d'instruction à l'usage des jeunes élèves des écoles primaires. Ouvrage contenant un précis sur l'origine de la Nation française et sa révolution; un exercice, en forme de Catéchisme, sur l'essence de notre gouvernement républicain; la Géographie de la République Française ; trois Tableaux par ordre alphabétique ; [...] les Droits de l'homme ; la Constitution ; un recueil des faits hérö̈ques les plus remarquables, par lesquels se sont signalés les Républicains Français ; une estampe représentant le jeune Barra au moment où il est assassiné, avec les faits hérö̈ques qui lui ont mérité les honneurs du Panthéon. Paris, Devaux - Patris, an II, 1 vol. in-12.

28. Code militaire, ou recueil méthodique des décrets relatifs aux troupes de ligne et à la gendarmerie nationale, rendus par les assemblées constituante et législative, et par la convention nationale, depuis 1789, jusques et compris le 15 juin 1793. Paris, Prault, 1793, 3 vol. in- $8^{\circ}$.

29. Rousseau, Jean-Jacques. Seconde partie des Confessions de J. J. Rousseau. Tome troisième. Deux éditions possibles : Genève, s. n., 1789, 1 vol. in- $8^{\circ}$; Paris, Poinçot - Lejay, 1789, 1 vol. in- $8^{\circ}$.

30. Marat, Jean-Paul. Les Chaînes de l'esclavage, Ouvrage destiné à développer les noirs attentats des princes contre les peuples; les ressords secrets, les ruses, les menées, les artifices, les coups d'état qu'ils emploient pour détruire la liberté et les scènes sanglantes qui accompagnent le despotisme. Paris, Imprimerie de Marat, l'an premier de la République, 1 vol. in $-8^{\circ}$.

31. Duquesnoy, Adrien Cyprien. Mémoire sur l'éducation des bêtes à laine et les moyens d'en améliorer l'espèce. Nancy, V ve Bachot, 1792, 1 vol. in- $8^{\circ}$.

32. Tableaux de la Révolution française, ou Collection de quarante-huit gravures, représentant les Evénemens principaux qui ont eu lieu en France depuis la transformation des Etats-Généraux en Assemblée nationale. Paris, Briffault de La Charprais, s. d., livraisons 20 à 25, 6 cahiers in-fol.

\section{Essai d'interprétation critique}

Préalablement à toute étude, il convient de soulever la question de l'identité du propriétaire de cette bibliothèque. Les auteurs qui se sont intéressés à l'inventaire ont tous considéré a priori qu'il répertoriait des livres du conventionnel Saint-Just. Mais la mention « ex-constituant » à la 
première ligne de l'inventaire amène à se demander si cette bibliothèque ne serait pas plutôt celle du constituant Fréteau de Saint-Just, guillotiné le 26 prairial an II. Un ensemble d'indices dont aucun ne serait suffisant à lui seul mais dont la conjonction est remarquable fait cependant écarter cette attribution. Les plus décisifs ${ }^{18}$ sont à notre avis la date à laquelle l'inventaire fut réalisé, qui suit d'un jour la vente des meubles, vêtements et autres biens que Saint-Just détenait dans son domicile de la rue Caumartin ${ }^{19}$, et la mention par l'inventaire des livraisons 20 à 25 des Tableaux de la Révolution française, publication périodique distribuée gratuitement aux députés à mesure de leur parution. Or les récents travaux de Claudette Hould ont montré que les numéros 20 à 25 des Tableaux sont parus à l'époque où Saint-Just siégeait à la Convention ${ }^{20}$.

La bibliothèque de Saint-Just est relativement modeste : elle compte soixante-treize volumes en tout, soit trois fois moins que celle de Robespierre et presque deux fois moins que celle de Couthon, par exemple ${ }^{21}$. Que Saint-Just ait eu à Paris moins de livres que Robespierre ou Couthon n'est d'ailleurs pas étonnant car, à la différence de ses aînés, il n'avait emménagé dans la capitale qu'en septembre 1792.

La part des livres en rapport avec la Révolution y est importante. L'inventaire ne décrit précisément que onze volumes relevant de cette catégorie, mais il faut leur ajouter les dix volumes du cinquième paquet pour lesquels est seulement noté qu'ils portent sur des « affaires du temps ». Cette formule courante dans les inventaires désigne en effet « les nouvelles de politique qui regardent la conjoncture présente, et la situation des choses du monde » (Dictionnaire universel de Furetière). Ainsi, c'est entre un quart et un tiers des ouvrages de la bibliothèque de Saint-Just qui concernent, de façons diverses, l'actualité révolutionnaire. Parmi ces

(18) Les autres tiennent à la place qu'occupent les œuvres de Montesquieu et de Rousseau, influences majeures du conventionnel, dans cette bibliothèque ainsi qu'à la présence d'un ouvrage de Marat, des trois premiers tomes du Code militaire et du tome XV des CEuvres complètes de Mably (pour tous ces livres, cf. la suite de notre développement).

(19) Archives de Paris, cote DQ ${ }^{10} 790$; publié partiellement par Gabriel VAUTHIER, Annales révolutionnaires, tome 15,1923 , p. 513-514.

(20) Claudette Hould, La Révolution par la gravure. Les Tableaux de la Révolution française, Vizille, Musée de la Révolution française - Paris, Réunion des Musées nationaux, 2002, p. 236. Claudette Hould date de façon moins précise les livraisons 24 à 26 des Tableaux qui, d'après ses recherches, pourraient avoir paru début 1794 ou après Thermidor. Les collections des Tableaux que possédaient Saint-Just et Couthon (Michel MoRINEAU, op. cit., p. 467) s'interrompant l'une et l'autre à la $25^{\mathrm{e}}$ livraison, on peut préciser que les $24^{\mathrm{e}}$ et $25^{\mathrm{e}}$ livraisons sont antérieures au 9 Thermidor.

(21) Cf. également « Les bibliothèques de quelques acteurs de la Révolution... » qui examine un corpus de vingt-six bibliothèques. La bibliothèque de Saint-Just est l'une des deux qui contient le moins de volumes (op. cit., p. 195). 
livres, le plus intéressant est certainement Les Chaînes de l'esclavage, traduction de l'ouvrage que Marat avait publié à Londres vingt ans plus tôt sous le titre The Chains of Slavery mais que son auteur a remanié pour la version française afin de le faire correspondre aux enjeux politiques contemporains. La présence de ce livre dans sa bibliothèque confirme le jugement très positif que Saint-Just porte sur les idées politiques de Marat dans ses discours. La bibliothèque de Saint-Just comprenait encore trois tomes d'un Code militaire réalisé à la demande du ministre de la Guerre Bouchotte, que le conventionnel a pu acquérir durant l'été 1793 alors qu'il travaillait à la section de la Guerre du Comité de salut public en coopération étroite avec ce ministre ${ }^{22}$, ainsi que Le Manuel des jeunes Républicains, compilation hétérogène et sans grand intérêt, surtout utile pour ses tableaux récapitulant les départements, districts, cantons et chefs-lieux. Dernier ouvrage mentionné dans l'inventaire, les Tableaux de la Révolution française ne sont pas significatifs dans la mesure où, comme nous l'avons dit, ce périodique était offert aux députés.

Quels pouvaient être les dix autres volumes en rapport avec l'actualité que l'inventaire ne décrit pas ? D'après les autres inventaires de révolutionnaires condamnés, il pourrait s'agir de publications en série tels que les procès-verbaux de la Convention, de volumes de la Collection générale des décrets rendus par l'Assemblée nationale, de journaux de format in $-8^{\circ}$ comme le Journal des débats et des décrets, d'ouvrages administratifs ou encore de recueils de textes imprimés plus ou moins hétérogènes ${ }^{23}$.

Après la Révolution française, c'est la philosophie du XVIII' siècle qui occupe le plus de place dans cette bibliothèque. Les auteurs les plus présents sont, sans surprise, Rousseau (huit volumes) et Montesquieu (sept volumes) dont on a souvent noté l'influence sur la pensée de Saint-Just ${ }^{24}$. Cependant, la composition de cette bibliothèque marque une différence dans la façon dont Saint-Just considérait chacun de ces auteurs : alors qu'il lisait Montesquieu dans une édition de ses Euvres complètes, il ne possédait que certaines des œuvres de Rousseau - Émile, le Contrat social dans une édition de 1791, une partie des Confessions - ainsi qu'une anthologie en deux volumes de ses œuvres. Saint-Just avait également chez lui un

(22) Jean-Pierre GRoss, Saint-Just, sa politique et ses missions, Paris, Bibliothèque nationale, 1976 , p. $52-55$.

(23) Les deux volumes présentés allusivement comme « Recueil de pieces affaires du temps » sont certainement des recueils factices de ce type.

(24) « Entre l'Esprit de la Révolution et l'Esprit des lois, la filiation est perceptible à la fois dans le titre, la présentation, la formulation et la manière d'aborder les grands problèmes, mais aussi dans les réponses » (Bernard VINOT, Saint-Just, Paris, Fayard, 1985, p. 114). 
recueil d'extraits classés par thèmes de David Hume. Le quinzième tome des Euvres de Mably, contenant les Principes de morale et un second texte attribué à l'abbé qui est un faux, serait quant à lui une acquisition tardive et en partie fortuite puisque, d'après une anecdote rapportée par Laurent de l'Ardèche ${ }^{25}$ qui la situe au printemps 1794, Saint-Just aurait emprunté le livre au Comité de salut public après une discussion entre ses membres. Sa pensée ne paraît en effet pas avoir été marquée par ce philosophe jusqu'à la rédaction des passages du Projet d'institutions consacré à la religion ${ }^{26}$.

Avec respectivement sept et deux volumes, l'histoire et le droit sont moins représentés dans cette bibliothèque. Saint-Just possédait une histoire générale : le Discours sur l'histoire universelle de Bossuet que complète une Suite anonyme, assez médiocre, résumant de façon chronologique les événements survenus entre 800 et 1700 principalement en France et en Europe. Saint-Just s'intéressait plus particulièrement à l'Angleterre et à la révolution anglaise, avec les trois tomes de La Vie d'Olivier Cromwel et les développements extraits de l'Histoire d'Angleterre que contient Le génie de M. Hume, ainsi qu'à l'histoire romaine. Sa bibliothèque contenait en effet l'ouvrage à succès que l'abbé de Vertot a consacré à la Rome antique, ainsi qu'un volume bilingue réunissant la Conjuration de Catilina et la Guerre de Jugurtha de Salluste. Les ouvrages de droit y sont remarquablement peu nombreux. Tout juste relève-t-on la Constitution de l'Angleterre de Jean-Louis de Lolme, autre succès de librairie du XVIII ${ }^{\mathrm{e}}$ siècle que l'on retrouve dans plusieurs bibliothèques de conventionnels condamnés, et un ouvrage de jurisprudence plus inattendu puisqu'il s'agit du Code des loix des Gentoux, une compilation réalisée à la demande de Warren Hastings pour instruire les colonisateurs anglais des règles et conventions des brahmanes.

Plusieurs tendances se dégagent des livres tant de philosophie que d'histoire et de droit décrits par l'inventaire. D'abord, un fort intérêt pour la philosophie morale et les questions politiques qui ne saurait étonner de la part de Saint-Just. Sa bibliothèque contenait les ouvrages majeurs sur la politique des philosophes des Lumières, et les livres d'histoire et de droit qui la composaient étaient tous propres à nourrir la réflexion d'un dirigeant politique. Il en est de même pour deux ouvrages plus anciens conservés dans sa bibliothèque qui sont des essais portant sur des aspects de la technique du gouvernement : Le Conseiller d'Estat généralement attribué

(25) Réfutation du livre de M. l'abbé de Montgaillard intitulé Histoire de France depuis la fin du règne de Louis XVI jusqu'en 1825, Paris, Delaforest, 1828, p. 331.

(26) Anne QUENNEDEY, « Le rôle de Saint-Just dans l'élaboration du décret du 18 floréal an II », à paraître. 
à Philippe de Béthune, et l'Essai sur les Mécontentemens populaires de William Temple, première partie des Euvres postumes du ministre de Charles II.

Par ailleurs, les lectures de Saint-Just montrent une attirance pour les penseurs anglo-saxons et pour l'Angleterre que l'on retrouve, parfois de façon plus prononcée, chez d'autres révolutionnaires ${ }^{27}$. Nous avons mentionné les ouvrages de Hume, Temple et de Lolme, ainsi que $L a$ Vie d'Olivier Cromwel; à ces ouvrages, il faut ajouter Les Chaînes de l'esclavage de Marat qui, outre ses nombreux exemples tirés de l'histoire anglaise, comporte en annexe un «Tableau des vices de la constitution angloise » développant des thèses contraires à celles que soutient de Lolme dans sa Constitution de l'Angleterre ${ }^{28}$.

Un autre centre d'intérêt que manifestent la bibliothèque de Saint-Just mais aussi les exemples historiques de ses discours, est l'Antiquité, particulièrement romaine. Le conventionnel possédait en effet la synthèse de l'abbé de Vertot, les deux ouvrages de Salluste, les Considérations sur les causes de la grandeur des Romains et de leur décadence contenues dans le tome VI des Euvres de Montesquieu, ainsi que des recueils de discours de Cicéron et de Démosthène dans des éditions en éclairant les enjeux historiques et politiques. Le quatrième livre du Contrat social contient aussi des chapitres sur les institutions romaines que sont les comices, le tribunat, la dictature et la censure.

Mais Saint-Just ne s'intéressait pas à la seule Antiquité gréco-romaine. Il possédait aussi des ouvrages traitant de la Chine ancienne, Yu le Grand et Confucius, et de l'Inde, le Code des loix des Gentoux, qui ont la particularité d'avoir été réunis en un seul volume ainsi que l'indique la précision «meme vol» après le titre Code des loix des Gentoux. Malgré leurs différences d'approche, ces deux ouvrages ont en effet en commun de porter sur des sociétés non européennes et précapitalistes généralement mal connues en Europe mais qui suscitaient au XVIII ${ }^{\mathrm{e}}$ siècle un fort intérêt dans une partie du public cultivé. Ils témoignent qu'existaient chez Saint-Just non seulement une curiosité

(27) Robespierre possédait ainsi des ouvrages de Locke, de Pope et de Bacon, The Guardian de Joseph Addison et Richard Steele, une Histoire de la Grande-Bretagne et une Grammaire anglaise (Fabienne RATINEAU, « Les livres de Robespierre au 9 Thermidor », AHRF, $\mathrm{n}^{\circ}$ 287, 1992, p. 132-134).

(28) Doit-on supposer que la connaissance qu'avait Saint-Just des penseurs anglo-saxons ait été beaucoup plus étendue que ce qu'en laisse connaître sa bibliothèque ? Dans l'Esprit de la Révolution, il s'étonne que Pope n'ait sa statue nulle part en Europe, critique dans Du Droit social les vues de Hobbes sur l'état de nature, et son discours du 29 novembre 1792 contient une référence à Adam Smith. Ce que Saint-Just dit d'eux n'incite cependant pas à penser qu'il connaissait ces auteurs de première main, comme c'est le cas pour Hobbes qu'il cite d'après Montesquieu. 
prononcée pour des civilisations très différentes de la nôtre mais aussi le désir de connaître précisément les mœurs et l'organisation politique et juridique d'autres peuples. Ce désir trouvait aussi à se satisfaire avec l'Introduction historique sur les Incas qui précède les Lettres péruviennes dans l'édition du livre de Madame de Graffigny que possédait Saint-Just et avec l'Esprit des loix ${ }^{29}$. En dépit de son sous-titre (« histoire chinoise »), Yu le Grand et Confucius est moins un ouvrage historique sur la Chine qu'un livre de philosophie pratique d'inspiration physiocratique portant sur les principes et l'action d'un bon gouvernement ${ }^{30}$. Plusieurs idées développées dans le Projet d'institutions de Saint-Just - le respect de la vieillesse, l'importance de l'amitié, la liberté des unions, l'institution des censeurs surveillant sévèrement les autorités constituées - pourraient avoir été en partie inspirées par la lecture de Yu le Grand et Confucius. Le Code des loix des Gentoux n'a en revanche pas d'écho dans les écrits de Saint-Just. Une allusion de l'Esprit de la Révolution ${ }^{31}$ laisse même penser qu'il a pu ne pas considérer d'un œil favorable les lois des brahmanes.

Si la bibliothèque de Saint-Just était peu fournie en livres d'histoire et de droit, elle contenait un nombre important d'ouvrages de littérature ${ }^{32}$ : avec treize volumes, cet ensemble constitue en effet la troisième catégorie de livres la mieux représentée après les ouvrages sur la Révolution et ceux de philosophie. On y trouvait les discours des deux orateurs qui, au XVIII siècle, sont regardés comme les plus grands orateurs ayant existé : Cicéron dans une édition bilingue, et Démosthène dans une traduction française parue sous la Révolution. Le corpus oratoire antique que Saint-Just avait à disposition à son domicile se trouvait complété par son exemplaire du Catilina et du Jugurtha de Salluste qui contient plusieurs harangues

(29) Cf. le grand nombre de développements sur la Chine et les Indes répertoriés par l'Index de l'Esprit des loix, par exemple.

(30) Concernant l'inspiration physiocratique de cet ouvrage rédigé pour servir à l'instruction du tsarévitch Paul I ${ }^{\mathrm{er}}$, on se reportera à Miguel ABENSOUR, « La philosophie politique de Saint-Just. Problématique et cadres sociaux », $A H R F, \mathrm{n}^{\circ} 185,1966$, p. 343-344. Sur l'idéalisation de la Chine par les physiocrates, voir Zhan SHI, «L'image de la Chine dans la pensée européenne du XVIII siècle : de l'apologie à la philosophie pratique », $A H R F, \mathrm{n}^{\circ} 347,2007$, p. 102-104.

(31) «Une des causes qui empêcheront la liberté de pénétrer dans les Indes, est la multitude des Bramines ; ce sont les rites qui enchaînent la plupart de ces pauvres peuples » (SAINT-Just, Euvres complètes, Anne KUPIEC et Miguel ABENSOUR (éd.), Paris, Gallimard, 2004, p. 419).

(32) Sur le sens moderne du mot littérature qui s'impose à partir des dernières décennies du $\mathrm{XVIII}^{\mathrm{e}}$ siècle et la révolution qui s'opère vers la même époque dans l'ancien système des Belles-Lettres, voir Jacques RANCIÈRE, La parole muette. Essai sur les contradictions de la littérature, Paris, Hachette, 1998. 
considérées elles aussi comme des modèles d'éloquence (harangues de Catilina, de César, de Caton, de Sylla ${ }^{33}$.

Parmi les ouvrages de littérature de sa bibliothèque parisienne, on remarque également trois œuvres majeures du XVII ${ }^{\mathrm{e}}$ siècle, les Fables de La Fontaine et Les Caractères de La Bruyère dans les éditions annotées de Pierre Coste, ainsi que Les Aventures de Télémaque de Fénelon. Les Aventures de Télémaque fut l'un des livres les plus lus jusqu' au $\mathrm{XX}^{\mathrm{e}}$ siècle, par la jeunesse notamment. Les lecteurs du XVIII ${ }^{\mathrm{e}}$ siècle paraissent avoir été particulièrement sensibles à deux aspects de l'œuvre : l'enseignement moral et politique de ce livre écrit pour exposer au duc de Bourgogne les qualités d'un grand roi et, peut-être plus encore, le charme poétique qui se dégage de ce texte. Pour cette raison, et bien qu'il soit écrit en prose, le Télémaque est pour les contemporains de Saint-Just moins un roman qu'une épopée dont les mérites littéraires sont comparés à l'Odyssée et à l'Enéide.

La littérature du XVII ${ }^{\mathrm{e}}$ siècle était donc assez bien représentée dans cette bibliothèque. Il ne semble pas qu'il en ait été de même pour le siècle suivant, puisque l'inventaire ne mentionne qu'une seule œuvre de fiction datant du XVIII ${ }^{\mathrm{e}}$ siècle, les Lettres d'une Péruvienne de Madame de Graffigny ${ }^{34}$. Les Lettres d'une Péruvienne sont pour l'essentiel un roman sentimental dans le goût du temps célébrant l'attachement indéfectible d'une femme malgré l'abandon de son amant. Sur cette trame se greffent des épisodes fortement inspirés des Lettres persanes, qui développent de façon convenue le thème du bon sauvage mis en contact avec la société européenne qui constate à quel point ses mœurs sont éloignées de la nature. Ce roman épistolaire eut un vif succès durant la seconde moitié du $\mathrm{XVIII}^{\mathrm{e}}$ siècle $^{35}$. Dernièrement, un courant universitaire a vu dans certaines des Lettres d'une Péruvienne l'expression d'un engagement en faveur d'une émancipation des femmes. Il n'est pas impossible que Saint-Just ait

(33) Ces trois éditions donnent aussi des indications sur la connaissance qu'avait Saint-Just des langues anciennes. Ses exemplaires de Cicéron et de Salluste étant bilingues, on peut supposer que Saint-Just était capable sinon de lire le latin dans le texte, du moins de goûter les qualités formelles de textes écrits dans cette langue; en revanche, qu'il n'ait pas cherché à se procurer Démosthène en grec donne à penser qu'il ne maîtrisait pas le grec ancien. Charles Vellay a publié une transcription de la liste des prix que Saint-Just reçut en classe de cinquième d'où il ressort qu'il commença à acquérir une aisance avec la langue latine durant ses années de collège ("Les premières luttes politiques de Saint-Just », La Revue de Paris, octobre 1906, p. 822).

(34) Saint-Just pouvait cependant lire des œuvres comme les Lettres persanes ou Le Temple de Gnide dans son exemplaire des Euvres complètes de Montesquieu.

(35) Daniel MORNET, « Les enseignements des bibliothèques privées (1750-1780) », Revue d'histoire littéraire de la France, t. 17, 1910, p. 449-496. 
été sensible au tableau de la condition misérable des femmes françaises qu'expose la trente-quatrième lettre.

Mais l'ouvrage de littérature le plus intéressant de sa bibliothèque est certainement la Jérusalem délivrée, le célèbre poème épique du Tasse, dans une édition de 1793. Sa présence dans la bibliothèque du conventionnel est à mettre en rapport avec le Télémaque de Fénelon qui, nous l'avons vu, était couramment considéré au XVIII ${ }^{\mathrm{e}}$ siècle comme une épopée en prose, avec Organt, l'épopée satirique en vers publiée par Saint-Just en 1789, ainsi qu'avec la phrase du Projet d'institutions énonçant que «Le prix de la poesie ne sera donné qu'à l'ode et a l'epopée $»^{36}$. Mise ainsi en perspective, l'acquisition tardive de cette édition du poème du Tasse montre que le conventionnel menait, les derniers mois de son existence, une réflexion sur les conditions de possibilité et l'utilité politique d'une poésie épique moderne dont la Jérusalem délivrée est considérée, tout au long du $\mathrm{XVIII}^{\mathrm{e}}$ siècle, comme le modèle ${ }^{37}$. Un dernier livre de littérature, l'Histoire poétique, qui est un exposé sur les mythes gréco-latins illustré d'extraits de poètes français des $\mathrm{XVII}^{\mathrm{e}}$ et $\mathrm{XVIII}^{\mathrm{e}}$ siècles, vient confirmer à la fois l'intérêt de Saint-Just pour la poésie d'inspiration élevée et un goût pour la mythologie antique que dénote également la présence du Télémaque dans sa bibliothèque.

Ainsi que Dominique Varry et Agnès Marcetteau-Paul l'ont indiqué ${ }^{38}$, Saint-Just fait partie des révolutionnaires dont la bibliothèque ne contenait pas de livre de religion. Pour autant, toute préoccupation religieuse ou en rapport avec la religion n'en est pas absente. Nous avons vu que Saint-Just avait chez lui l'Émile, qui contient la Profession de foi du vicaire savoyard ; il possédait aussi l'édition dite de Port-Royal des Pensées de Pascal, dans laquelle la plupart des chapitres sont ordonnés autour de questions religieuses, et le Discours sur l'histoire universelle qui interprète l'Histoire d'après l'action supposée de la Providence divine. Saint-Just a pu encore lire dans le Contrat social ainsi que dans le faux Mably du tome XV de ses Euvres complètes des développements sur les rapports que l'État doit entretenir avec la religion.

(36) BNF, NAF 24136, feuillet 17.

(37) Saint-Just pourrait avoir eu un intérêt ancien pour l'ouvrage du Tasse : le premier feuillet du recto du carnet du manuscrit $D u$ Droit social porte en effet le titre biffé « Jerusalem Poëme » tracé d'une écriture maladroite qui semble enfantine. Il n'est néanmoins pas certain que ce soit celle du jeune Saint-Just (Anne QUENNEDEY, « Note philologique sur le manuscrit de Saint-Just faussement intitulé De la nature », AHRF, $\mathrm{n}^{\circ} 351,2008$, p. 126-127).

(38) Agnès Marcetteau-PaUl et Dominique VArRY, op. cit., p. 204. 
Enfin, la bibliothèque de Saint-Just contenait deux ouvrages qui ne relèvent pas des catégories sous lesquelles nous avons rangé ses autres livres. Le premier est un manuel scolaire de mathématiques acquis par Saint-Just après 1788 , alors que ses études secondaires étaient achevées. S'agissaitil d'un achat utile, destiné à rafraîchir ou compléter les compétences mathématiques qu'il était susceptible d'utiliser en tant que législateur ? Dans la mesure où le contenu du manuel de l'abbé Mazéas excède de beaucoup les besoins de Saint-Just dans ses fonctions à la Convention nationale puis au Comité de salut public, on doit plutôt supposer qu'il a souhaité approfondir pour son propre compte une matière bénéficiant alors d'un large engouement ${ }^{39}$. Les manuels de l'époque insistent en particulier sur les qualités de rigueur logique que les mathématiques développent, y compris chez les jeunes gens ne se destinant pas aux sciences.

L'autre ouvrage témoignant de la diversité des centres d'intérêt de Saint-Just durant les années où il siège à la Convention est un livre sur l'élevage des moutons intitulé Mémoire sur l'éducation des bêtes à laine et les moyens d'en améliorer l'espèce ${ }^{40}$. On peut s'étonner que les charges politiques qui étaient alors les siennes lui aient laissé le loisir de se plonger dans la lecture des détails techniques qu'énumère cet ouvrage. Cependant, son discours sur les subsistances du 29 novembre $1792^{41}$ et, en 1794, le Projet d'institutions montrent que Saint-Just se préoccupait des moyens d'accroître le cheptel français, et en particulier le cheptel ovin : les feuillets 25 et 35 du manuscrit des Institutions prévoient en effet des institutions ayant cette fin.

\section{Limites de l'inventaire et devenir des livres de Saint-Just après fructidor an III}

L'apport principal de l'inventaire de la bibliothèque parisienne de Saint-Just est de mettre en lumière des lectures que l'on ne soupçonnerait pas en son absence et qui ont pu jouer un rôle dans la formation de ses idées, comme Yu le Grand et Confucius, les Euvres postumes de William

(39) L'abbé Bossut écrit par exemple que les mathématiques « ont acquis parmi nous, surtout depuis quelques années, un degré de faveur, qui a prodigieusement multiplié les Livres élémentaires destinés à les expliquer et à les répandre » (Cours de mathématique, Paris, Claude-Antoine Jombert, 1775 , p. IX).

(40) Son auteur Adrien Cyprien Duquesnoy, ancien constituant puis maire de Nancy, fut destitué en août 1793 après que l'ouverture de l'armoire de fer eut dévoilé ses rapports avec le roi. Incarcéré à La Force en 1794, Duquesnoy fut libéré après Thermidor (Edna Hindie LEMAY, Dictionnaire des Constituants, Paris, Universitas, t. I, 1991, p. 321-322).

(41) SAINT-JUST, Euvres complètes, op. cit., p. 494-495. 
Temple, Le génie de M. Hume et les Pensées de Pascal. Il a aussi l'utilité de montrer l'importance de centres d'intérêt (pour les sociétés précapitalistes non européennes, pour la pensée politique de Marat, pour l'épopée) que ses discours ne manifestent pas, à eux seuls, aussi clairement.

Mais, comme on devait s'y attendre, cet inventaire ne rend pas compte de toutes les lectures du conventionnel ${ }^{42}$. En particulier, l'inventaire ne fait pas état de plusieurs ouvrages dont les œuvres de Saint-Just portent la trace, ni d'auteurs que certains de ses contemporains, parfois sujets à caution, affirment qu'il a lus. C'est ainsi qu'Augustin Lejeune, une connaissance soissonnaise qu'il fit employer au Bureau de police générale, assure que Saint-Just fut un lecteur assidu d'Homère et de Racine durant ses jeunes années, et qu'il lut l'abbé Raynal pendant la Révolution ${ }^{43}$. Élisabeth Le Bas a, quant à elle, raconté dans ses souvenirs qu'en octobre 1793, dans la voiture qui les emmenait en Alsace, son mari et Saint-Just lisaient à haute voix « pour passer le temps [...] des pièces de Molière ou quelques passages de Rabelais $»^{44}$.

Parmi les auteurs que Saint-Just cite dans ses ouvrages et que l'inventaire de l'an III ne mentionne pas, on remarque plusieurs poètes dont Chaulieu (1639-1720), auteur de poèmes anacréontiques, et le satiriste Gilbert (1750-1780) $)^{45}$. On s'étonne aussi de ne pas trouver mention dans l'inventaire d'ouvrages de théâtre, alors que Saint-Just avait pour la poésie dramatique un fort intérêt qui lui fit écrire la comédie en vers Arlequin-Diogène et une épigramme en rapport avec une tragédie de Le Belloy $^{46}$.

Le grand absent de la bibliothèque de Saint-Just est cependant Voltaire. Son absence est d'autant plus frappante que ses œuvres se trouvent dans de nombreuses bibliothèques d'acteurs de la Révolution dans d'imposantes éditions en quarante, soixante-dix ou quatre-vingt-douze

(42) On prendra néanmoins garde à ne pas surestimer l'étendue des lectures de Saint-Just : lorsque celui-ci fait référence à Ulpien et à Aulu-Gelle, par exemple, dans Du Droit social, c'est en se fondant sur ce que Montesquieu a mentionné de leurs ouvrages dans l'Esprit des loix.

(43) Notice historique sur Saint-Just publiée par Alfred BÉGIS dans : Saint-Just et les bureaux de la police générale au Comité de salut public en 1794, Paris, Les Amis des livres, 1896, p. 30. Une phrase ambiguë que Lejeune prête à Saint-Just pourrait indiquer que le conventionnel avait aussi apprécié, dans sa première jeunesse, les Idylles du poète suisse Gessner (ibidem, p. 33).

(44) StÉFAnE-Pol (Paul CoUtANT), Le Conventionnel Le Bas d'après des documents inédits et les Mémoires de sa veuve, Paris, Flammarion, 1901, p. 132.

(45) SAINT-JUST, Euvres complètes, op. cit., p. 265 (citation de Chaulieu). Cette édition oublie l'épigraphe anonyme d'Organt qui, ainsi que l'a vu Bernard Vinot, est tirée du poème de Gilbert Le Dix-huitième Siècle (Bernard VinOT, op. cit., p. 66).

(46) Bernard VINOT, op. cit., p. 49. 
volumes $^{47}$, et qu'il est clair que Saint-Just connaissait très bien plusieurs ouvrages de Voltaire qu'il imite ou auxquels il fait des emprunts dans Organt. De la même façon, les livres de Rousseau de sa bibliothèque ne sont pas les seules œuvres de lui que Saint-Just ait lues, puisqu'on trouve dans ses discours des phrases démarquées d'ouvrages moins connus de cet auteur. Que des livres avec lesquels Saint-Just avait une évidente familiarité manquent dans l'inventaire pourrait s'expliquer par la volonté d'avoir avec soi à Paris une bibliothèque réduite qui soit essentiellement une bibliothèque de travail, pour laquelle il n'était donc pas utile de retenir des ouvrages mal adaptés à la situation politique ouverte par le 10 Août.

Après le 13 fructidor an III, les livres qui composaient la bibliothèque de Saint-Just furent transférés du dépôt de la rue Cerutti à celui de la rue Saint-Marc ${ }^{48}$ puis au dépôt de la rue de Lille ${ }^{49}$. S'il n'existe pas de document indiquant ce qu'ils devinrent par la suite, les travaux de Jean-Baptiste Labiche $^{50}$ ont établi que l'ensemble des ouvrages entreposés dans le dépôt rue de Lille furent transportés en l'an $\mathrm{V}$ au dépôt de la rue des Cordeliers, dans lequel des prélèvements en vue d'enrichir les fonds des bibliothèques publiques furent organisés, puis en l'an XIII à l'hôtel de Chabrillant, rue de l'Université, où les prélèvements se poursuivirent jusqu'à la réunion à la bibliothèque de l'Arsenal des derniers restes des dépôts littéraires de la Révolution, en 1811.

C'est ainsi que la bibliothèque de Saint-Just, qui avait été conservée intacte dans des paquets étiquetés pendant les deux années précédant son arrivée rue des Cordeliers, se trouva divisée à la suite des opérations de triage menées dans les dépôts littéraires afin de compléter les collections des quatre grandes bibliothèques publiques parisiennes (Bibliothèque nationale et bibliothèques de l'Arsenal, du Panthéon et des Quatre-Nations), des bibliothèques des départements ainsi que de plusieurs bibliothèques spécialisées. Nous avons cherché à savoir s'il serait possible de retrouver dans les bibliothèques publiques françaises des livres ayant appartenu à Saint-Just. Bien qu'aléatoire, cette entreprise ne paraissait pas en effet vouée à l'échec dans la mesure où les livres ayant appartenu au conventionnel

(47) Agnès Marcetteau-PAUl et Dominique VARRY, op. cit., p. 200.

(48) Inscription manuscrite en haut à gauche de l'inventaire.

(49) Inventaire des dépôts littéraires, Bibliothèque de l'Arsenal, tome $1, \mathrm{~ms} \mathrm{n}^{\circ} 6487$. Ils portaient le numéro 85 et étaient entreposés au rez-de-chaussée.

(50) Jean-Baptiste LABICHE, Notices sur les dépôts littéraires et la révolution bibliographique de la fin du dernier siècle, d'après les manuscrits de la bibliothèque de l'Arsenal, Paris, A. Parent, 1880 . 
pourraient porter des marques distinctives (ex-libris, reliure particulière, etc.) ou avoir été versés en grand nombre dans un même fonds.

Cette recherche a permis de trouver à la Bibliothèque nationale le premier livre décrit dans l'inventaire ${ }^{51}$ : le volume contenant sous une même reliure Yu le Grand et Confucius par Nicolas Gabriel Clerc (Soissons, P. Courtois, 1769, 1 vol. in- $4^{\circ}$ ) et le Code des loix des Gentoux par Nathaniel Brassey Halhed (Paris, Stoupe, 1778, 1 vol. in- $4^{\circ}$ ). Plusieurs indices conduisent en effet à affirmer que le livre de la Bibliothèque nationale de France conservé sous la cote Fb-18111-18112 ne saurait être que l'exemplaire ayant appartenu à Saint-Just. D' abord, la relative rareté des deux ouvrages ${ }^{52}$; comme ils n'ont de surcroît entre eux qu'un rapport ténu, qu'existent plusieurs exemplaires de ces deux ouvrages reliés ensemble parait improbable. Ensuite, la reliure du livre de la Bibliothèque nationale coté Fb-18111-18112, est bien d'époque XVIII ${ }^{\mathrm{e}}$ siècle. Enfin, le fait que ce livre provienne effectivement des dépôts littéraires révolutionnaires.

Le livre de la Bibliothèque nationale est un fort volume in $-4^{\circ}$ de vingt centimètres de large sur vingt-six de haut pour une épaisseur de huit centimètres et demi, relié pleine peau en veau marbré. Sans être précieuse, la reliure est assez caractéristique : les plats sont simplement décorés d'un triple filet d'encadrement, mais les compartiments du dos à cinq nerfs sont abondamment ornés de fleurons et de palettes dorés, avec des pièces de titre rapportées de maroquin bordeaux, pour « Yu le Grand et Confucius », et fauve, pour « Code des Gentoux ». Les trois tranches sont teintes en rouge, les gardes sont de papier marbré rouge, vert, jaune et blanc à motifs de coquille, les tranchefiles sont de mêmes couleurs et le signet est un ruban vert. Hormis les inscriptions portées par les bibliothécaires en début et en fin de volume, nous n'avons pas remarqué d'annotations. Le livre n'a pas non plus d'ex-libris.

Comme l'indiquent le cachet « Bibliothèque du roi - Fontainebleau » ajouté sous la Restauration sur la page de titre et plusieurs tampons d'inventaire ${ }^{53}$, cet ouvrage a appartenu à la bibliothèque impériale du palais de Fontainebleau avant d'être versé dans le fonds de la Bibliothèque nationale de France où lui fut attribuée son actuelle cote en « $\mathrm{Fb}$ », pour «Fontainebleau ». Avant d'être au palais de Fontainebleau, ce livre de

(51) « yu le grand et confucius par clerc deux tomes 1 vol, codes des loix des Jentoux meme vol».

(52) Le Catalogue collectif de France recense dix-neuf exemplaires de Yu le Grand et treize du Code des loix des Gentoux.

(53) Inventaires de 1856 et 1873. 
Saint-Just passa par la bibliothèque du Conseil d'État : Yu le Grand... et le Code des loix des Gentoux sont en effet répertoriés dans le catalogue de cette bibliothèque réalisé en $1803^{54}$. La bibliothèque du Conseil d'État a deux origines : les dépôts littéraires révolutionnaires et la bibliothèque $\mathrm{du}$ Directoire, elle-même formée en puisant dans les dépôts littéraires de Paris et Versailles, qui lui fut donnée en $1800^{55}$. Ainsi, le volume de Saint-Just contenant Yu le Grand... et le Code des loix des Gentoux quitta le dépôt des Cordeliers soit entre 1798 et 1800 pour la bibliothèque du Directoire exécutif, avant d'être transférée en 1800 à la bibliothèque du Conseil d'État, soit entre 1800 et 1803 , pour entrer directement dans cette bibliothèque ${ }^{56}$.

En 1807, l'ouvrage fut transporté au palais de Fontainebleau avec l'ensemble des livres du Conseil d'État, Napoléon ayant demandé qu'une bibliothèque soit constituée dans ce palais. Lorsqu'en 1888 la bibliothèque de Fontainebleau, qui comptait alors plus de 43000 volumes, fut supprimée comme les autres bibliothèques des palais impériaux, elle fut rattachée à la Bibliothèque nationale de France. Seul un dixième des livres du fonds Fontainebleau a été effectivement transporté dans les locaux de la Bibliothèque nationale. Notre exemplaire fait partie des livres portant les cotes $\mathrm{Fb}-17597$ à Fb-21113 qui y furent transférés à la fin du XIX ${ }^{\mathrm{e}}$ siècle $^{57}$.

C'est ainsi qu'avant d'achever sa course à la Bibliothèque nationale, le livre de Saint-Just contenant Yu le Grand... et le Code des loix des Gentoux passa par quatre dépôts et trois bibliothèques, et ne subit pas moins de

(54) Catalogue des livres de la bibliothèque du Conseil d'État, Paris, Imprimerie de la République, an XI, t. I, p. 107, n' 1123, et t. II, p. 276, n 8956. D'après le catalogue et une mention à l'encre sur la page de garde de l'exemplaire Fb-18111-18112 de la Bibliothèque nationale, le volume de la bibliothèque du Conseil d'État était rangé sous la désignation « Recueil n 18 ». En l'an XI, cette bibliothèque comptait approximativement 25000 volumes.

(55) Anne-Marie RABANT, « Barbier et la bibliothèque du Conseil d'État », Bulletin d'information de l'Association des Bibliothécaires français, $n^{\circ} 57,1967$, p. 243-248. La bibliothèque du Directoire se trouvait hôtel de Croy, rue du Regard, et contenait environ 30000 volumes.

(56) C'est Antoine-Alexandre Barbier, successivement conservateur de la bibliothèque du Directoire, bibliothécaire du Conseil d'État puis bibliothécaire des palais impériaux, qui s'occupa du choix des livres à puiser dans les dépôts pour ces bibliothèques et qui fut également chargé de faire transporter à Fontainebleau les livres du Conseil d'État («Barbier et la bibliothèque du Conseil d'État », op. cit., et Maurice LECOMTE, « Les bibliothèques et les bibliothécaires du palais de Fontainebleau depuis le XVIII ${ }^{\mathrm{e}}$ siècle », dans Mélanges historiques sur Fontainebleau, Fontainebleau, Maurice Bourges, 1904, p. 72-77). Auparavant, ce bibliographe réputé avait été membre de la Commission temporaire des arts et chargé de répartir entre les différentes bibliothèques de Paris les livres de dépôts, fonction qui lui avait apportée une bonne connaissance de ce fonds gigantesque.

(57) Les cotes Fb-17597 à Fb-21113 correspondent à un ensemble de recueils de pièces reliées que les bibliothécaires n'ont pas voulu dérelier (Eugène Gabriel LEDOS, Histoire des catalogues des livres imprimés de la Bibliothèque nationale, Paris, Éditions des Bibliothèques nationales, 1936, p. 217). Depuis son déménagement sur le site de Tolbiac, ils y sont conservés réunis au département Philosophie, histoire, sciences de l'homme (Bibliothèque nationale de France, Dictionnaire électronique des fonds spéciaux, entrée « Fontainebleau»). 
dix déménagements ${ }^{58}$. D'autres livres de la bibliothèque de Saint- Just connurent-ils le même sort que ce volume en étant, comme lui, affectés à la bibliothèque du Conseil d'État puis à celle de Fontainebleau ? Seuls trois livres répertoriés par le Catalogue des livres de la bibliothèque du Conseil d'État et l'inventaire de la bibliothèque du palais de Fontainebleau ${ }^{59}$ pourraient éventuellement correspondre à ceux décrits par l'inventaire du 13 fructidor an III. En conséquence, sauf à supposer que Bonaparte, Cambacérès et Lebrun, qui s'adjugèrent plusieurs milliers de volumes de la bibliothèque du Directoire ${ }^{60}$, aient récupéré une grande partie des livres qui avaient composé la bibliothèque de Saint-Just, on doit considérer que les ouvrages du conventionnel n'ont pas été versés en nombre dans la bibliothèque du Conseil d'État. Le plus probable est qu'une dispersion générale des livres de Saint-Just entre les différentes bibliothèques de Paris et de province ait eu lieu entre l'an $\mathrm{V}$ et 1811, des ouvrages ayant pu être vendus ou détruits ${ }^{61}$, particulièrement lorsqu'ils étaient brochés ou incomplets.

Louise AMPILOVA-TUIL maîtrise d'histoire louise.tuil@voila.fr

Catherine Gosselin docteure d'État en droit rigambert@dbmail.com

Anne QUENNEDEY docteure en littérature française, agrégée de lettres modernes quennedey.anne@wanadoo.fr

(58) D'abord aménagée dans la chapelle Saint-Saturnin, la bibliothèque de Fontainebleau fut installée après 1851 dans la Galerie de Diane. La bibliothèque du Conseil d'État fut successivement aux Tuileries puis au Louvre.

(59) Fichier manuscrit établi en 1899, conservé à la Bibliothèque nationale de France (Tolbiac). Il convient de consulter le fichier du fonds Fontainebleau car les prélèvements dans les dépôts pour la bibliothèque du Conseil d'État se sont poursuivis après le catalogage de 1803.

(60) « Barbier et la bibliothèque du Conseil d'État », op. cit., p. 243.

(61) Sur les ventes et destructions des livres des dépôts, voir Jean-Baptiste LABICHE, op. cit., p. $51-55$ et p. $117-118$. 